\title{
Agnès Fine, Françoise-Romaine Ouellette (dir.), Le Nom dans les sociétés occidentales contemporaines
}

Toulouse, Presses universitaires du Mirail, 2005, 252 pages.

\section{Danièle Voldman}

\section{(2) OpenEdition}

\section{Journals}

Édition électronique

URL : http://journals.openedition.org/clio/7531

DOI : 10.4000/clio.7531

ISSN : $1777-5299$

Éditeur

Belin

Édition imprimée

Date de publication : 1 janvier 2008

Pagination : 257-258

ISBN : 978-2-85816-973-3

ISSN : 1252-7017

Référence électronique

Danièle Voldman, « Agnès Fine, Françoise-Romaine Ouellette (dir.), Le Nom dans les sociétés occidentales contemporaines », Clio. Histoire, femmes et sociétés [En ligne], 27 | 2008, mis en ligne le 06 août 2008, consulté le 21 septembre 2020. URL : http://journals.openedition.org/clio/7531 ; DOI : https://doi.org/10.4000/clio.7531

Ce document a été généré automatiquement le 21 septembre 2020.

Tous droits réservés 


\title{
Agnès Fine, Françoise-Romaine Ouellette (dir.), Le Nom dans les sociétés occidentales contemporaines
}

Toulouse, Presses universitaires du Mirail, 2005, 252 pages.

\author{
Danièle Voldman
}

1 Accompagnée d'une aspiration générale à une plus grande égalité entre les sexes et d'une remise en cause de l'autorité paternelle, le dernier tiers $d u x^{e}$ siècle a été une période de transformation profonde et rapide des organisations familiales traditionnelles. Alors que les familles étaient en partie structurées autour des pratiques séculaires de dénomination, neuf contributions franco-québécoises explorent les modalités d'un système en mutation. Il faut d'abord souligner la triple originalité de ce recueil : l'aspect comparatif des pratiques de dénomination en France et au Québec, deux sociétés occidentales francophones développées; l'analyse d'une évolution dont on ne connaît pas encore toutes les conséquences; la contribution d'anthropologues ordinairement mieux outillés pour étudier les permanences que les changements. Ensuite, on doit apprécier le subtil va-et-vient des auteurs entre l'affirmation de la puissance symbolique du système onomastique et la description des mille et une formes de ses contournements, qu'il s'agisse du nom ou du prénom, accolés ou pris séparément.

2 La puissance symbolique du système est rappelée par plusieurs articles à travers l'étonnante persistance de la croyance dans l'obligation légale de la perte du nom des femmes mariées au profit de celui de leurs maris. C'est ainsi que malgré des transformations législatives fondamentales qui ont permis à "quelques sociétés occidentales comme l'Allemagne, la Suède, l'Espagne, le Portugal ainsi que le Québec et d'autres provinces canadiennes [de rendre] possible la transmission du nom de famille de la mère parmi les noms de famille donnés à l'enfant ", la transmission du nom du père reste un choix prédominant. Alors que les mères ont des stratégies diversifiées quant à leur propre appellation (nom de jeune fille, nom de l'époux, nom double avec variation de l'ordre dans lequel les noms sont déclinés), le nom du père est largement le 
choix préféré pour les enfants, même en cas de nom double où celui de la mère suit presque toujours celui du père.

Cette conclusion conduit à l'une des constatations essentielles de l'ouvrage: sous l'apparente diversité des pratiques et leur totale dérégulation, « la nouvelle manière de donner les prénoms et les noms » révèle des continuités dans les traditions familiales mais aussi nationales et religieuses. Certes, aujourd'hui, le rôle des parents est primordial dans le choix du prénom et du nom de l'enfant. Il semble sacrifier d'abord à l'individualisme, à des modes plus ou moins fantaisistes comme les prénoms des familles des grands feuilletons télévisés ou à la recherche de l'originalité. De fait, le prénom désigne avant tout « un individu à part entière dès sa naissance, objet du désir du couple " plutôt qu'un parent disparu ou un saint, servant d'exemple et de patron. Pourtant, les analyses fouillées concernant les deuxième et troisième prénoms cherchent à montrer des permanences traditionnelles. Encore celles-ci intéressentelles avant tout les familles bien établies et non marginales. De ce point de vue, les pratiques des couples mixtes sont riches d'enseignement. Résultat de lutte pour la prééminence d'une culture en Europe ou d'un compromis comme au Canada, le choix des prénoms des enfants des couples mixtes révèle toujours un projet identitaire: montrer et légitimer les origines multiples ou au contraire les nier au profit d'une intégration onomastique, pratique que l'on retrouve dans le choix des noms d'enfants orphelins, abandonnés ou adoptés.

4 Ainsi, alors que tous les auteurs soulignent la dérégulation du système de dénomination, ils tentent néanmoins d'en repérer des significations. Si tout nom est héritage, il est logique que dans un monde qui tend à s'homogénéiser, les traditions s'affaiblissent et soient plus difficiles à suivre. En ce qui concerne le nom, elles résistent cependant à la sécularisation de l'état-civil tout en prenant des chemins détournés. Cela ne facilite pas le travail d'élucidation. Quels indicateurs sociaux ou culturels peuvent receler des façons de faire mouvantes après des siècles d'apparente stabilité ? Si on a parfois l'impression que les auteurs n'ont pas vraiment trouvé ce qu'ils cherchaient, conduisant à des conclusions aussi prudentes que nuancées (par exemple en ce qui concerne les noms mixtes ou les enjeux religieux de la transmission des prénoms), la lecture de cet ouvrage donne des clefs pour comprendre des pratiques foisonnantes qui suivent plus qu'on ne croit des injonctions enracinées dans le passé. 